\title{
Environmental Bioindication using Some Morphometric and Physiological Parameters of Plants
}

\author{
Guzel R Valeeva*, Zarina M Khafizova and Violetta A Gabdrakhimova \\ Department of Institute of Ecology and Environmental Management, Federal University, Russian
}

*Corresponding author: Guzel R Valeeva, Department of Institute of Ecology and Environmental Management, Federal University, Russian.

To Cite This Article: Guzel R Valeeva, Environmental Bioindication using Some Morphometric and Physiological Parameters of Plants. Am J Biomed Sci \& Res. 2019 - 4(1). AJBSR.MS.ID.000748. DOI: 10.34297/AJBSR.2019.04.000748

Received: July 09, 2019 | Published: July 15, 2019

\section{Introduction}

The work aims at assessing the quality of the environment in the city of Kazan (Russian Federation) through the study of such indicators as the fluctuating asymmetry (FA), the chlorophyll content, and the delayed chlorophyll fluorescence in the leaves of birch (Betula pendula). We collected the samples at 21 sites within the city with high levels of anthropogenic stress. In the study, we evaluated the morphometric parameters of the leaf lamina (on the basis of these parameters, we later determined the level of fluctuating asymmetry), the content of photosynthetic pigments in the leaves, as well as the delayed chlorophyll fluorescence and the acetone extract from the leaves. According to data obtained by using the Spearman's rank correlation coefficient, we found a statistically significant inverse correlation between the fluctuating asymmetry and the delayed-fluorescence average intensity measured for the leaves in low-light mode (DFlow).

Additionally, using the Shapiro-Wilk criterion, we found that the content of carotenoids in the leaves is normally distributed. For this reason, we employed nonparametric methods for statistical data processing.

\begin{tabular}{|c|c|c|}
\hline Parameter & Conditionally Dirty & Conditionally Clean \\
\hline FA & Value above & Value below \\
\hline $\mathrm{DF}_{0}$ & Value below & Value below Values above \\
\hline $\mathrm{DF}_{\text {max }}$ & Value below & Value above \\
\hline DFvariable & Value above & Value below \\
\hline DFlow & Value below & Value above \\
\hline DFhigh/DFlow & Value below & Value above \\
\hline
\end{tabular}

\begin{tabular}{|c|c|c|c|c|}
\hline \multicolumn{7}{|l|}{ Table 2. } \\
\hline Pollution level & FA -95.000\% & FA $\mathbf{+ 9 5 . 0 0 0 \%} \%$ & DF0 -95.000\% & DF0 +95.000\% \\
\hline Conditionally dirty & 0,065587 & 0,077739 & $83,94,949$ & $8,67,776$ \\
\hline Conditionally clean & 0,061123 & 0,063422 & $95,93,551$ & 1006,76 \\
\hline Pollution level & DFmax -95.000\% & DFmax +95.000\% & DFvariable +95.000\% & DFvariable +95.000\% \\
\hline Conditionally dirty & 3320,88 & $34,21,391$ & 0,742335 & 0,749998 \\
\hline Conditionally clean & $34,62,614$ & $35,63,515$ & 0,713851 & 0,725572 \\
\hline Pollution level & DFlow $\mathbf{- 9 5 . 0 0 0} \%$ & DFlow +95.000\% & DFhigh/DFlow -95.000\% & DFhigh/DFlow +95.000\% \\
\hline Conditionally dirty & $18,53,571$ & $19,67,866$ & 0,389182 & 0,451672 \\
\hline Conditionally clean & 2252,35 & $23,96,855$ & 0,310869 & 0,353131 \\
\hline
\end{tabular}


The Mann-Whitney method is a statistical criterion used to estimate the differences between two independent samples. Thus, all fluctuating asymmetry values were divided into two groups: "conditionally clean" (from FA $=0.056$ to $\mathrm{FA}=0.065$ ) and "conditionally dirty" (FA > 0.065). We found statistically significant differences between these two groups (Table 1). We also noted a higher content of chlorophyll A and carotenoids in the leaves from the conditionally clean zone. The difference, however, between the samples is not statistically significant in this case. The values obtained are given in Table 2. Here we show the intervals that contain the "conditionally clean" and "conditionally dirty" groups at a 95 percent confidence level.

The obtained intervals of delayed fluorescence values can later ease the assessment of the environmental condition. Indeed, the FA measurement is a quite laborious process, whereas the delayed fluorescence of leaves requires only about 5 minutes to be measured (depending on the given number of cycles of measurement). We can thus use the delayed-fluorescence data to predict into which of the two pollution levels we should include a given site of the city. If data do not fall into any of the two intervals considered or more accurate forecasts are required, then a comprehensive analysis is necessary. The obtained intervals of delayed fluorescence values can later ease the assessment of the environmental condition. Indeed, the FA measurement is a quite laborious process, whereas the delayed fluorescence of leaves requires only about 5 minutes to be measured (depending on the given number of cycles of measurement). We can thus use the delayed-fluorescence data to predict into which of the two pollution levels we should include a given site of the city. If data do not fall into any of the two intervals considered or more accurate forecasts are required, then a comprehensive analysis is necessary.

Keywords: Chlorophyll; Delayed fluorescence; Environmental monitoring; Pollution; Bioindicator; Fluctuating asymmetry; Birch 